\title{
Instability and freezing in a solidifying melt conduit
}

14 rises before freezing. Previous theoretical models of a tube driven by a constant pressure

15 drop suggest that once the pressure minimum is reached, the states for a lower flux

16 should be unstable and the tube should therefore freeze up. In our experiments, flux and

17 pressure drop were coupled, and this motivates us to extend the theory for low-Reynolds

18 number flow through a tube with solidification to incorporate a simple pressure drop-flux

19 relationship. Our model predicts a steady-state relationship between flux and pressure

20 drop that has a minimum of the pressure as the flux is varied. The stability properties of

21 these steady states depend on the boundary conditions: for a fixed flux, they are all stable,

22 whereas for fixed pressure drop, only those with a flux larger than that at the pressure 
23 drop minimum are stable. For a mixed pressure-flux condition, the stability threshold of

24 the steady states lies between these two end members. This provides a possible

25 mechanism for the experimental observations.

\section{Introduction}

27 Injected liquids that freeze as they flow are common in many areas of engineering

28 (injection molding, freezing, metallurgy) as well as in earth and planetary sciences (lava

29 tubes, magma conduits, glaciology, and magma fissure flows). In such cases, liquid flows

30 through a region whose boundary temperature is below the solidification temperature of

31 the liquid, so that advection of heat by the warm liquid acts in tandem with removal of

32 heat by the boundary. In some cases, the cooling is weak enough that solid may form at

33 the boundary but leave a central melted tube where liquid flows. In other cases the entire

34 body of liquid may freeze so that all flow ceases. It is useful to know the conditions that

35 are necessary for such freezing.

36 In the geophysical literature, the pioneering study of the dynamics of melting and

37 solidifying material was for flow up a fissure with variable gap width [Bruce and

38 Huppert, 1989, 1990], where conditions for melt-back (widening) or solidification

39 (narrowing) of the gap are calculated from thermal energy budgets. This was followed by

40 many studies of the dynamics of either fissure flow or lava dynamics, investigating

41 situations such as the temperature distribution and velocity profile in a magma tube, or

42 the driving pressure required to keep it open [e.g. Sakimoto and Zuber, 1998, Dragoni et

43 al., 2002, Sakimoto and Gregg, 2001, Klingelhofer et al., 1999]. These studies invariably

44 use simplified, time-independent geometries for the tube boundary, and generally, little

45 analysis has been made of the stability of the flows. A notable exception is the theoretical 
46 study by Lister and Dellar, [1996], in which the cooling occurs at infinity and therefore

47 no steady-state tube is possible.

$48 \quad$ For engineering purposes, numerous studies focus on flow of a liquid in a

49 container whose walls are below the freezing temperature. Applications include injection

50 molding, the freezing of water, the condensation of water vapor in ducts, and metal

51 casting, among others. For example, experiments with water demonstrate the focusing of

52 flow into a narrow region along with the formation of waves of solid on the walls, and in

53 some cases freeze-up [Zerkle and Sunderland, 1968 Mulligan and Jones, 1976 Hirata

54 and Ishihara 1985, Weigand et al., 1997]. A common feature is that the curve of steady-

55 state pressure drop against flux exhibits sizeable curvature, in many cases reaching a

56 minimum such that as the flux is gradually decreased, the pressure drop first decreases,

57 then increases, a result that has been recovered in theoretical studies [Zerkle and

58 Sunderland, 1968, Lee and Zerkle, 1969.] If the flow is driven by imposing a fixed

59 pressure drop, however, the low-flux branch of this curve, where pressure drop increases

60 with decreasing flux, is unstable: a perturbation making a smaller cross sectional area

61 produces more drag, which produces slower flow that leads to colder liquid and more

62 solidification and finally to total freezing [Sampson and Gibson, 1981, Richardson 1985].

63 If, instead, the flow is driven by a pump imposing a fixed flux, the steady state is

64 presumed to be stable, although a complete stability analysis has never been done; a

65 smaller cross sectional area makes a faster flow that brings warmer fluid from upstream

66 to the region, which widens the perturbation. The constant flux upstream condition is

67 widely used in theories that calculate the solid accumulation along flow ducts of assorted

68 material properties and shapes [e.g., Mulligan and Jones, 1976, Epstein and Chueng, 
69 1983, Richardson, 1986], but such problems do not exhibit flow freeze-up from an

70 instability.

71 Since theory shows that stability depends on the particular type of flow boundary

72 condition that is imposed at the upstream end, our attention here is focused upon the

73 stability of solidifying flow with a more general upstream condition than either constant

74 flux or constant pressure drop. We tackle the question of stability with both experiment

75 and theory. First, we describe laboratory experiments of flow through a pipe whose

76 temperature is held below the solidus, in which there was a coupling between flux and

77 pressure drop (Section 2). The flow froze when the steady-state flux was below a certain

78 value. As the steady-state flux was decreased in successive experiments to this value, the

79 pressure drop across the tube reached a minimum and then increased before freeze-up.

80 This result is not explained by either constant flux or constant pressure drop models, one

81 of which suggests freeze-up should never occur, and the other that it should occur as soon

82 as the pressure minimum is reached. It motivates us to investigate the stability of low-

83 Reynolds number flow through a tube using a standard idealized theoretical model with

84 the addition of a mixed pressure-flux upstream driving condition (Section 3). Essentially,

85 we suppose the tube drains from an upstream reservoir into which fluid is pumped at a

86 constant rate, so the total amount of fluid in the reservoir determines the driving pressure

87 and therefore the flux through the tube. Naturally, this new upstream condition is

88 intended to be a more realistic model both of conditions in our experiment as well as in

89 some types of geological melt conduits, and possibly in some engineering applications. A

90 linear stability analysis shows that the mixed upstream condition allows the stable range

91 of flow to extend to lower values of flux that are unstable for fixed pressure drop. Thus, it 
92 is in qualitative accord with the laboratory results. In addition, the theory predicts an

93 oscillatory instability that has not been found in previous theoretical studies. Numerical

94 simulations recover both new features (Section 3.4). In Section 3.5 we show how the

95 basic model (without the stability results) can be used straightforwardly to provide a

96 realistic constraint on the length of geological melt conduits.

97 The central implication of these results is that stability is very sensitive to the 98 upstream conditions that drive the melt through the tube. This sensitivity may be one

99 mechanism behind the complex nature of many real solidifying flows in nature and

100 industry.

\section{Experiments with freezing of flow through a tube.}

104 is shown in Figure 1. The pipe was a standard glass condenser for a chemistry laboratory 105 with a central glass pipe of radius $r_{0}=0.49 \times 10^{-3} \mathrm{~m}$ surrounded by a sleeve (see Table 1

106 for list of symbols). The length of the portion of the pipe surrounded by this sleeve was

$107 L=0.18 \mathrm{~m}$. The sleeve was flushed by water from a constant temperature bath at

108 temperature $T_{0}$ that was accurate to $\pm 0.1{ }^{0} \mathrm{C}$. The central axis of the condenser was

109 placed horizontally. Liquid at $20{ }^{\circ} \mathrm{C}$ was fed from a constant displacement metered

110 pump into one end of the condenser. The pump volume flux rate (henceforth simply

111 called either flux or, in case of a pump setting, the pumping rate) was calibrated to $\pm 2 \%$.

112 The other end was the tube exit fitted with a rubber stopper with a flat notch cut along the

113 top. The liquid exited the glass tube by flowing over this notch; therefore, the stopper 
114 served as a miniature dam so that the pipe within the condenser remained filled with

115 liquid at all times with no air traveling upstream from the exit into the tube. A photograph

116 of the outlet with the stopper removed after a run shows a circular drainage channel

117 surrounded by solid (Figure 2). The ridges in the solid are evidence of uneven

118 solidification whose origin will not be studied further here. The liquid was 1-Octadecene

119 (Chevron Phillips C18, kindly donated),. In this study, we simply call this material a

120 wax. The freezing point (solidus temperature) is $T_{s}=17.8^{\circ} \mathrm{C}$ and the pour point is half a

121 degree higher at $18.3^{\circ} \mathrm{C}$, indicating that viscosity increases greatly close to the solidus.

122 The specifications for the liquid state are: a thermal conductivity of $k=0.114 \mathrm{~W} / \mathrm{m}^{0} \mathrm{~K}$, a

123 specific heat of $c_{p}=2.26 \times 10^{3} \mathrm{j} / \mathrm{kg}{ }^{0} \mathrm{~K}$ with significant changes in value near freezing

124 temperature (Bundhu at al. 1998), a density of $\rho=785 \mathrm{~kg} / \mathrm{m}^{3}$ (these three give a thermal

125 diffusivity of $\kappa=0.64 \times 10^{-7} \mathrm{~m}^{2} \mathrm{~s}^{-1}$ ) and kinematic viscosity values of $v=8.28 \times 10^{-6}$

$126 \mathrm{~m}^{2} \mathrm{~s}^{-1}$ at $31^{0} \mathrm{C}$ and $v=3.8 \times 10^{-6} \mathrm{~m}^{2} \mathrm{~s}^{-1}$ at $37.8^{0} \mathrm{C}$. Also, the fluid is very hygroscopic.

127 Since the model developed in subsequent sections assumes constant material properties,

128 the fact that viscosity and specific heat changes greatly in the temperature range of

129 interest means that we will only be able to compare the experimental results with

130 prediction qualitatively.

131 For all experiments, the temperature of the liquid pumped into the condenser was

$132 T_{i}=20^{\circ} \mathrm{C}$. After starting the liquid pump, the temperature of the water flushing the

133 sleeve was set to a value below the solidus so that the wax became solid along the inner

134 radius of the glass pipe as sketched in Figure 1, with flow occurring in a central liquid

135 tube. The liquid tube radius varied in the flow direction and it was a function of the

136 pumping rate and sleeve temperature. We measured pressure immediately upstream of 
137 the condenser by splitting the upstream plastic tubing with a Y connection. The tube in

138 one side of the $\mathrm{Y}$ was the input to the condenser and the other plastic tube was held

139 vertically next to a centimeter scale to allow a measurement of pressure of the upstream

140 fluid. Since pressure at the downstream end was fixed at atmospheric pressure, the

141 elevation of the liquid surface in the vertical plastic tube above the elevation of the outlet

142 was proportional to pressure drop across the condenser. This elevation was read to a

143 precision of $1 \mathrm{~mm}$. The vertical tube is also a storage region for liquid supplied by the

144 pump. In fact, the difference between the flux of the pump and the flux out through the

145 condenser is proportional to the rate of change of height in the vertical pressure tube.

146 This provides a mixed pressure-flux upstream boundary condition to the flow through the

147 condenser. The exact expression for this will be derived in the next section.

148 The top of the vertical plastic tube was bent over and extended back to the wax

149 reservoir as an overflow. If upstream pressure became too great, the overflowing liquid

150 indicated freeze-up of the tube

151 The procedure for these experiments at the beginning of each day was to start

152 with everything at room temperature so the wax was completely liquid. A run

153 commenced by turning on the wax pump to a desired pumping rate and then changing the

154 cold bath temperature from $20{ }^{\circ} \mathrm{C}$ to the desired value, which we call $T_{0}$. After about 15

155 minutes, the wax solidified along the inner radius of the tube and the flow continued

156 through the liquid tube. The elevation in the vertical tube was measured many times until

157 the value was steady, and then the final value of pressure (in units of vertical elevation)

158 was recorded. The flux was also measured then. 
Figure 3 shows the elevation of the liquid surface in the vertical tube versus the

160 imposed pumping rate, or flux for many runs in experiments with cold bath temperatures

161 set to two different values: $T_{0}=5.0{ }^{\circ} \mathrm{C}$ and $T_{0}=10.0{ }^{\circ} \mathrm{C}$. At both temperatures the

162 flowing liquid froze shut at a pumping rate approximately $5 \%$ below the measurement on

163 the extreme left. To the right of the freezing point, the inverse relation between the

164 pressure and pumping rate was unmistakable. For $T_{0}=10.0{ }^{0} \mathrm{C}$, pressure increased

165 slightly with pumping rate for flux $Q_{i}>0.5 \times 10^{-6} \mathrm{~m}^{3} \mathrm{~s}^{-1}$ but for $T_{0}=5.0{ }^{0} \mathrm{C}$, a pressure

166 increase with flux is not visible. The errors for the pressure measurement and for the

167 calibration of the pumping rate are approximately the size of the symbols. Since

168 obviously the scatter about a smooth curve for all the data is considerable, we concluded

169 after careful checking that the scatter is not from errors in measurement. In addition, we

170 conducted long runs to determine whether the scatter was due to the experiment duration

171 being too short. For all these experiments (which were conducted for more than two

172 hours each, and compromise $70 \%$ of the data points), such scatter persisted even though

173 the pressure reading had been constant for the entire second hour. Therefore, we believe

174 the scatter is a basic feature and the scatter might possibly be due to small differences in

175 the detailed shape of each frozen solid. In support of this, Figure 2 shows irregularities in

176 the solid surface near the exit.

177 The experiment results are scaled by noting that the experimental flow tube has

178 the following variables: the glass tube radius $r_{0}$, tube length $L$, fluid viscosity $\mu$, fluid

179 density $\rho$, fluid thermal diffusivity $\kappa$, temperature at the inlet $T_{i}$, temperature of the

180 surface of the tube $T_{0}$, temperature of solidus $T_{s}$, and flux of the liquid initially entering

181 upstream $Q_{i}$. This totals 9 variables with four units: temperature, force, length and time. 
182 Therefore, five dimensionless numbers are needed. Two of them are simply temperature

183 ratios, but they are best combined and expressed as $T_{n}=\frac{T_{s}-T_{0}}{T_{i}-T_{s}}$. A third is aspect ratio

184 of the tube $r_{0} / L$. A fourth is Prandtl number $\operatorname{Pr}=v / \kappa$, and the last is nondimensional

185 flux $q_{i}=\frac{2}{\kappa \pi L} Q_{i}$. In addition, we calculate a value of nondimensional pressure drop

$186 \Delta P=\frac{r_{0}^{4}}{4 \mu \kappa L^{2}} P$, where $P$ is the pressure above atmosphere pressure at the upstream end

188 Using the values for this liquid, the Prandtl number is $\operatorname{Pr}=129$. Using the tube

189 length and radius, and using the magnitude for flux near the minimum of about

$190 Q_{i}=0.3 \times 10^{-6} \mathrm{~m}^{3} \mathrm{~s}^{-1}$ from Figure 3, we get $q_{i}=15$. The magnitude of scaled pressure

191 from the same figure is found using the hydrostatic equation for pressure $P=\rho g H$,

192 where acceleration from gravity is $g$ and a typical elevation of wax in the vertical

193 pressure measuring tube is $H=0.02 \mathrm{~m}$. From this, we get $\Delta P=1650$.

194 Next, the values of actual critical fluxs for freezing were checked by four precise

195 experiments at four different values of $T_{0}=2.5,5.0,7.5$, and $10.0{ }^{0} \mathrm{C}$. For each of these

196 values, an experimental run started with the pump set at a value that allowed continuous

197 flow. Then, the freezing point was approached by decreasing the pumping rate by 5\%

198 increments and waiting an hour or more to see if the flow froze. If the flow did not freeze

199 after that time interval, another decrease was made. The aggregate time for each run was

200 many hours. The lowest values of pumping rate at the above four temperature settings are

$2010.42,0.23,0.18$, and $0.16 \times 10^{-6} \mathrm{~m}^{3} \mathrm{~s}^{-1}$, successively, These correspond to non-

202 dimensional values of $q_{i}=23.2,12.7,9.95$, and 8.84 at $T_{n}=6.95,5.82,4.68$, and 3.55, 
203 respectively. Flow ceased by freezing shut for incrementally changed pumping rates that

204 were approximately $5 \%$ below these rates.

205 In experiments using more than the 5\% incremental decrease in pumping rate

206 from one experiment to the next, the critical flux for freezing was measurably larger. For

207 example, the wax always froze shut for experiments at $T_{0}=10{ }^{\circ} \mathrm{C}$ with a steady pumping

208 rate of $0.36 \times 10^{-6} \mathrm{~m}^{3} \mathrm{~s}^{-1}$ and then after steady flow developed were given a $33 \%$ decrease

209 in pumping rate to $0.24 \times 10^{-6} \mathrm{~m}^{3} \mathrm{~s}^{-1}\left(q_{i}=19.89\right.$ to 13.26$)$, The exact reason why a large

210 incremental decrease leads to a higher critical flux than the value with a $5 \%$ incremental

211 decrease, which in this case is $0.16 \times 10^{-6} \mathrm{~m}^{3} \mathrm{~s}^{-1}\left(q_{i}=8.84\right)$, is unknown. Possibly the

212 upstream pressure cannot build up rapidly enough to allow sufficient flux through the

213 melt region when the interior radius shrinks.

214 After a steady flow developed, the stopper at the exit was removed to view the

215 inner conduit radius by looking into the end of the pipe. A light beam from a slide

216 projector at a right angle to the tube and directed at the end of the tube far from the

217 camera illuminated fluid upstream as the white circle in Figure 2. Regrettably, we are

218 skeptical of using such images to attempt to measure the diameter of the liquid conduit.

219 Clearly, there was large distortion of the light as it passed to the camera across the curved

220 liquid/air surface. Also, each light beam arriving to the camera from the inside of the

221 liquid tube was bent by the axial temperature distribution within the liquid tube with the

222 axial equivalent of the mirage effect. Therefore, no optical measurements of the tube

223 radius as a function of flow rate and sleeve temperature were attempted.

224 If the flux and the bath temperature were slightly above the values that gave

225 freezing, the flow was easily made to freeze even with very small disturbances. For 
226 example, with a sleeve temperature of $2.5,{ }^{0} \mathrm{C}$ and pumping rate of $0.42 \times 10^{-6} \mathrm{~mm}^{3} \mathrm{~s}^{-1}$,

227 when the pump was stopped for five seconds, the flow ceased and never started again.

228 Conversely, with the same initial conditions the flow resumed most of the time if the

229 pump was stopped for three seconds, and it always resumed if the pump was stopped for

230 only one second. We also found that a piece of very fine copper wire inserted into the

231 liquid hole readily nucleated a freezing event.

\section{Flow through a tube, theory}

\subsection{Fundamental Equations}

234 We begin the analysis by reviewing a standard theoretical model for a melt conduit of

235 flow at low Reynolds number into a long cold pipe [eg Zerkle and Sunderland, 1968].

236 The pipe has a fixed length $L$ in the $x$-direction and it has a perfectly circular cross-

237 section with constant radius $r_{0}$ (Figure 4). Liquid enters the pipe at a uniform initial hot

238 temperature $T_{i}$ and it flows with laminar flow. The boundary of the pipe is maintained at

239 a constant temperature $T_{0}$ that is colder than the solidification temperature $T_{s}$. The

240 temperature varies continuously from $T=T(0, x, t)>T_{s}$ in the liquid at the center of the

241 tube, to $T_{0}$ at $r_{0}$. Solid material forms a tube of radius $a(x, t)$ at the isotherm $T=T_{s}$.

242 A number of assumptions are made to make the model analytically tractable. A full

243 list can be found in Zerkle and Sunderland [1968], but we mention those that will be

244 most important. First, the basic flow is made as simple as possible by assuming that there

245 are constant material properties, a simple cutoff solidification temperature, and no

246 buoyancy force. Second, the Reynolds number is small enough for there to be no

247 turbulence and no inertia in the momentum equation. Third, the length $L$ is assumed to be

248 large enough compared to $r_{0}$ that changes in the along-tube direction $x$ are slow. Finally, 
249 the Stefan number is assumed to be large, so that the solidification process and

250 corresponding motion of the crust are much slower than the thermal, advective, or

251 viscous timescales. Therefore time-derivatives are only retained in the equation for the

252 radius, and while other fluid variables are time-dependent, they are only quasi-steadily so

253 via their dependence on the radius. We now proceed to introduce the basic equations.

254 The velocity in the downstream direction is given by the well-known equation for

255 flow at low Reynolds number [eg Turcotte and Schubert, 2002], $\frac{\partial P}{\partial x}=\mu \frac{1}{r} \frac{\partial}{\partial r}\left(r \frac{\partial u}{\partial r}\right)$,

256 where $\partial P / \partial x$ is pressure gradient in the axial direction, $u$ is velocity in the axial direction,

$257 \mu$ is fluid viscosity and $r$ is the radial coordinate. The radial velocity $v$ can be found

258 from the condition of non-divergence, and is non-zero because the radius of the tube

259 changes in the flow direction. The solution for $u$ with the boundary condition $u=0$ at

$260 r=a(x, t)$ is Poiseuille flow $u=-\frac{\partial P}{\partial x} \frac{\left(a^{2}-r^{2}\right)}{4 \mu}$ Integrating over the area determines the

261 flux $Q$ whose relation to the pressure gradient is

$262 \quad \frac{\partial P}{\partial x}=-\frac{8 \mu Q}{\pi a^{4}}$,

263 so the velocity can also be written as

$\left.264 \quad u=\frac{2 Q}{\pi a^{2}}(1-(r / a))^{2}\right)$.

In the solid, the temperature field $T_{e}$ satisfies a diffusion equation when the $x$ -

266 derivatives and time-derivatives are neglected: 


$$
\frac{1}{r} \frac{\partial}{\partial r}\left(r \frac{\partial T_{e}}{\partial r}\right)=0
$$

268 with the boundary conditions $\left.T_{e}\right|_{r=r_{0}}=T_{0},\left.\quad T_{e}\right|_{r=a}=T_{s}$. This can be solved to give

$$
T_{e}=\frac{T_{0}-T_{S}}{\ln \frac{r_{0}}{a}} \ln \frac{r}{a}+T_{S} .
$$

$$
u \frac{\partial T}{\partial x}+v \frac{\partial T}{\partial r}=\kappa \frac{1}{r} \frac{\partial}{\partial r}\left(r \frac{\partial T}{\partial r}\right)
$$

273 with boundary conditions $T(r=a)=T_{S}, \quad T(x=0)=T_{i},\left.\quad \frac{\partial T}{\partial r}\right|_{r=0}=0$

274 It is more convenient to solve this by defining a new variable $\eta=r / a$, which scales the 275 radial coordinate by the radius of the tube, so that streamlines of the flow are lines of

276 constant $\eta$. Under this transformation equation (3.5) becomes

$$
\frac{2 Q}{\kappa \pi a^{2}}\left(1-\eta^{2}\right) \frac{\partial T}{\partial x}=\frac{1}{a^{2}} \frac{1}{\eta} \frac{\partial}{\partial \eta}\left(\eta \frac{\partial T}{\partial \eta}\right)
$$

$$
\text { with boundary conditions }\left.T\right|_{\eta=1}=T_{s},\left.\quad \frac{\partial T}{\partial \eta}\right|_{\eta=0}=0,\left.\quad T\right|_{x=0}=T_{i} \text {. }
$$


282 a standard Stefan equation [e.g. Turcotte and Schubert, 2002]

283

284

$$
\frac{L_{H}}{c_{p}} \frac{\partial a}{\partial t}=\kappa\left(\left.\frac{\partial T_{e}}{\partial r}\right|_{r=a}-\left.\frac{\partial T}{\partial r}\right|_{r=a}\right)
$$

286 where $\kappa$ is thermal diffusivity of both the liquid and solid, which are assumed here to be 287 equal in magnitude, $L_{H}$ is the latent heat of solidification, and $c_{p}$ is the heat capacity of 288 the liquid. The rate of change of the radius of the tube is proportional to the difference in 289 heat flux at the boundary of the tube, which, by the slowly-varying-in- $x$ assumption, is 290 the flux in the radial direction only.

\subsection{Steady-state Solutions}

We first consider the solution for the steady-state of the model, given by the

294 steady components of $(3.1,3.3,3.6,3.7)$ with the corresponding boundary conditions.

295 The equations are non-dimensionalized with $x=L \chi, \quad a=r_{0} \alpha, \frac{T-T_{s}}{T_{i}-T_{s}}=\theta$, $296 \frac{T_{e}-T_{s}}{T_{i}-T_{s}}=\theta_{e} \quad Q=\frac{\kappa L \pi}{2} q, \quad P=\frac{4 \mu \kappa L^{2}}{r_{0}^{4}} p, \quad$ and $\quad u=\frac{\kappa L}{r_{0}^{2}} u^{\prime} \quad$. Pressure is non-

297 dimensionalized so it remains in the balance to first order, and flux is non298 dimensionalized so that the effect of conductive cooling is balanced by advection. The 299 model depends on a dimensionless imposed temperature difference 
300

$$
T_{n}=\frac{T_{s}-T_{0}}{T_{i}-T_{S}}
$$

$$
u^{\prime}=\frac{q}{\alpha^{2}}\left(1-\eta^{2}\right) \quad, \quad \theta_{e}=\frac{T_{n} \ln \eta}{\ln \alpha} \quad(\eta \geq 1)
$$

304 and the pressure drop across the tube $\Delta p$ is related to the flux by:

305

306

$$
\Delta p=q \int_{0}^{1} \frac{1}{\alpha^{4}} d \chi
$$

311 This can be solved by separation of variables to give

$$
q\left(1-\eta^{2}\right) \frac{\partial \theta}{\partial \chi}=\frac{1}{\eta} \frac{\partial}{\partial \eta}\left(\eta \frac{\partial \theta}{\partial \eta}\right)
$$

313 where $\lambda_{n}, \phi_{n}$ are the eigenvalues and eigenfunctions of the problem

$314 \frac{1}{\eta} \frac{\partial}{\partial \eta}\left(\eta \frac{\partial \phi_{n}}{\partial \eta}\right)+\lambda_{n}^{2}\left(1-\eta^{2}\right) \phi_{n}=0, \quad \phi_{n}(0)=1, \phi_{n}(1)=0, \phi_{n}^{\prime}(0)=0$. The solution was

315 originally found by Graetz [1883] for flow of uniform viscosity through a pipe of

316 constant radius, and was modified for steady flow with solidification as in this

317 configuration by Zerkle and Sunderland [1968]. The $A_{n}$ are constants determined from 
318 the upstream temperature distribution. A more complete discussion of this solution,

319 including numerical values, is given in the appendix of Sakimoto and Zuber [1998]. In

320 steady-state, the dimensionless equation at the liquid solid interface becomes

$$
\left.\frac{\partial \theta}{\partial \eta}\right|_{\eta=1}=\left.\frac{\partial \theta_{e}}{\partial \eta}\right|_{\eta=1}
$$

$$
\left.\frac{\partial \theta_{e}}{\partial \eta}\right|_{\eta=1}=\frac{T_{n}}{\ln \alpha}
$$

$$
\left.\frac{\partial \theta}{\partial \eta}\right|_{\eta=1}=\sum G_{n} e^{-\lambda_{n}^{2} \chi / q}, \quad \text { where } \quad G_{n}=\left.A_{n} \frac{\partial \phi_{n}}{\partial \eta}\right|_{\eta=1}
$$

329 so the radius of a steady-state tube is

$$
\alpha(\chi)=\exp \left(\frac{T_{n}}{\sum G_{n} e^{-\lambda_{n}^{2} \chi / q}}\right)
$$

332 Profiles of $\alpha$ for several different values of $q$ are shown in Figure 5a. Note the

333 relation between $\alpha, q$, and $\Delta p$. If flux $q$ is prescribed then (3.14) gives an explicit

334 solution for $\alpha$, while if $\Delta p$ is prescribed it must be solved in conjunction with (3.10),

335 which provides a transcendental integro-differential equation for $\alpha$. Figure 6 a shows the 
336 pressure drop as a function of flux for a steady-state tube, for a particular choice of

337 temperature constant. This has a minimum $\Delta p_{c}$ at a critical flux $q_{c}$, suggesting that when

$338 \Delta p>\Delta p_{c}$ there are two solutions for a steady-state tube and when $\Delta p<\Delta p_{c}$ there are no

339 possible tubes, a fact which has been verified analytically in [Holmes, 2007]. The critical

340 pressure drop $\Delta p_{c}\left(T_{n}\right)$ and critical flux at which it is attained $q_{c}\left(T_{n}\right)$ are shown in Figure

$3415 b$.

$342 \quad$ Note the qualitative similarities between the analytic pressure drop-flux

343 relationship in Figure 6a and the experimental results in Figure 3: as flux is decreased

344 there is a very weak decline in pressure drop, and then a sudden sharp increase for low

345 values of flux.

348 To investigate stability we introduce an upstream condition with an additional

349 parameter to capture each of three possibilities: (i) constant flux, (ii) constant pressure,

350 and (iii) a model allowing the two variables to co-vary. One assumes that the tube is fed

351 from an upstream reservoir that in turn is fed by a steady volume flux of rate $Q_{i}$. (The

352 model can also be derived by assuming the upstream reservoir is elastic.) Flow from the

353 reservoir obeys the equation

354

355

$$
A \frac{d H}{d t}=Q_{i}-Q
$$


357 where $A$ is the cross-sectional area of the reservoir and $H$ is fluid elevation in it. The

358 downstream end of the tube is open and hence at atmospheric pressure, so the pressure

359 drop across the tube is given by

360

$$
\Delta P=g \rho H
$$

361

362

Letting the timescale be $S r_{0}^{2} / \kappa$, where Stefan number is $S=L_{H} / c_{p}\left(T_{i}-T_{S}\right)$, and

363 non-dimensionalizing the other scales as before, leads to the non-dimensional system

364

365

366

$$
\frac{\partial \alpha}{\partial t}=\frac{1}{\alpha}(E(\alpha)-I(\chi, q))
$$

367

$$
\frac{d \Delta p}{d t}=\tau\left(q_{i}-q\right)
$$

368 where the temperature gradient in the solid at the solid-liquid interface is

$369 E(\alpha)=\left.\frac{\partial \theta_{e}}{\partial \eta}\right|_{\eta=1}=\frac{T_{n}}{\ln \alpha}$, and the temperature gradient in the liquid at the interface is

$$
\Delta p=q \int_{0}^{1} \frac{1}{\alpha^{4}} d \chi
$$

$I(\chi, q)=\left.\frac{\partial \theta}{\partial \eta}\right|_{\eta=1}=\sum G_{n} e^{-\lambda_{n}^{2} \chi / q}$

371 This model has a new non-dimensional parameter $\tau=\frac{\pi g S r_{0}^{6}}{8 A v \kappa L}$, which measures the rate

372 of change of the upstream pressure relative to the rate of change of the radius of the

373 interface, and is proportional to the Stefan number times a thermal response time $r_{0}^{2} / \kappa$ 
374 divided by the hydraulic reservoir response time $A L v / g r_{0}^{4}$. The latter is the exponential

375 time for a viscous fluid to empty the reservoir with no solidification $\left(T_{n} \rightarrow 0\right)$.

376 The model also depends on the non-dimensional flux $q_{i}$ into the upstream

377 reservoir. Therefore, the dynamics of (3.15) are determined by the three parameters

$378 T_{n}, \tau, q_{i}$. When $\tau \ll 1$, the elevation, or pressure in the reservoir adjusts extremely slowly

379 to changes in the flux, and by extension the radius of the tube, so the system should

380 behave as if the pressure drop were held constant, with a constant-pressure-drop system

381 recovered exactly when $\tau=0$. When $\tau \gg 1$, the pressure in the reservoir adjusts rapidly

382 to the flux into the reservoir so the system should behave as if the flux through the tube

383 were held constant. Thus, setting different values of $\tau$ allows us to quantitatively

384 interpolate between constant flux and constant pressure drop conditions.

385 Let us now examine the linear stability of (3.15). Expanding to first order in small

$386 \varepsilon, q=q_{0}+\varepsilon q_{1}, \alpha=\alpha_{0}+\varepsilon \alpha_{1}$, and $\Delta p=p_{0}+\varepsilon p_{1}$ (note we have dropped the $\Delta$ symbol

387 for the pressure drop steady-states and perturbations), the steady-state is

388

389

390

$$
q_{0}=q_{i}
$$

391

$$
\alpha_{0}=\alpha_{0}\left(\chi, q_{i}\right)=\exp \left(\frac{T_{n}}{\sum G_{n} e^{-\lambda_{n}^{2} x / q_{i}}}\right)
$$

$$
p_{0}=q_{i} \int_{0}^{1} \frac{1}{\alpha_{0}^{4}} d \chi
$$

393 and the $O(\varepsilon)$ parts are 


$$
\frac{\partial \alpha_{1}}{\partial t}=\frac{1}{\alpha_{0}}\left(\left.\frac{d E}{d \alpha}\right|_{\alpha_{0}} \alpha_{1}-\left.\frac{\partial I}{\partial q}\right|_{q_{0}} q_{1}\right)
$$

$$
\frac{d p_{1}}{d t}=-\tau q_{1}
$$

$$
q_{1}=\frac{p_{1}-\left.\frac{\delta \Delta p}{\delta \alpha}\right|_{\alpha_{0}, q_{0}}\left[\alpha_{1}\right]}{\left.\frac{\partial \Delta p}{\partial q}\right|_{\alpha_{0}, q_{0}}}
$$

400 have taken care to distinguish between partial derivatives and functional derivatives, by 401 using the symbol $\partial$ for a partial derivative and $\delta$ for a functional derivative, which

402 results in a linear operator. We simplify notation by writing $\left.E_{\alpha}(\chi) \equiv \frac{1}{\alpha_{0}} \frac{d E}{d \alpha}\right|_{\alpha_{0}}$. Let us

403 analyze the three different boundary conditions in turn.

404 Case (i): constant flux. The stability of the constant flux case is simple to 405 analyze separately. Replacing (3.17b) with the condition $q_{1}=0$ and substituting for $406 E(\alpha)$, equation $(3.17 \mathrm{a})$ becomes

$$
\frac{d \alpha_{1}}{d t}=E_{\alpha} \alpha_{1}=\frac{-T_{n} \alpha_{1}}{\alpha_{0}^{2} \ln ^{2} \alpha_{0}}
$$

410 Since both $\alpha_{0}, T_{n}>0$, we have that $\operatorname{sgn}\left(d \alpha_{1} / d t\right)=-\operatorname{sgn}\left(\alpha_{1}\right)$ for every $\chi$, so this 411 equation is sign-definite and hence linearly stable. 
413 Case (ii): constant pressure. This case was first analyzed by Sampson and

414 Gibson, [1981]. Recall that for a given pressure drop there are two possible steady-state

415 tubes, one with $q>q_{c}$ and one with $q<q_{c}$, where $q_{c}$ is the value of flux which

416 minimizes pressure drop. By computing the single eigenvalue in the discrete spectrum of

417 the operator on the RHS of (3.17), Sampson and Gibson showed that only the former is

418 linearly stable. Holmes [2007] analyzed this case in more detail by considering the full

419 spectrum of the operator, obtaining the same results for the discrete spectrum and further

420 showing that the continuous spectrum is exactly Range $\left\{E_{\alpha}\right\}=(-\infty, c)$ where $c<0$, so

421 that only the discrete spectrum determines the stability properties.

422

423 Case (iii): variable pressure and flux. This case is considerably more difficult to

424 analyze analytically, and we will ultimately rely on numerical results. These show that as

425 in the constant pressure case, the continuous spectrum appears to be Range $\left\{E_{\alpha}\right\}$ which

426 is entirely negative, so we focus our analysis on the discrete spectrum.

427 Returning to (3.17), the equations can be rewritten by noting that $\alpha_{0}$ solves the

428 equation $E\left(\alpha_{0}(q, \chi)\right)=I(q, \chi)$, so taking the partial $q$-derivative and evaluating at

$429 q_{0}$ gives $\left.\frac{\partial I}{\partial q}\right|_{q_{0}}=\left.\left.\frac{d E}{d \alpha}\right|_{\alpha_{0}} \frac{\partial \alpha_{0}}{\partial q}\right|_{q_{0}}$. Here we introduce symbols $A_{q}, P_{q}, P_{\alpha}\left[\alpha_{1}\right]$ to represent the

430 derivative terms, which are defined precisely in Table 2. Under these transformations,

431 (3.17a) and (3.17b) become

432 
433

$$
\frac{d \alpha_{1}}{d t}=E_{\alpha}\left(\alpha_{1}-\frac{p_{1}-P_{\alpha}\left[\alpha_{1}\right]}{P_{q}} A_{q}\right)
$$

434

$$
\frac{d p_{1}}{d t}=\frac{-\tau}{P_{q}}\left(p_{1}-P_{\alpha}\left[\alpha_{1}\right]\right)
$$

438 form $\left(\alpha_{1}, p_{1}\right)=e^{\lambda t}\left(\tilde{\alpha}_{1}, \tilde{p}_{1}\right)$, substitute into the above equations, and solve to get

$$
\tilde{p}_{1}=\frac{\tau P_{\alpha}\left[\tilde{\alpha}_{1}\right]}{\left(P_{q} \lambda+\tau\right)}
$$

$$
\tilde{\alpha}_{1}=\frac{-E_{\alpha}\left(\tilde{p}_{1}-P_{\alpha}\left[\tilde{\alpha}_{1}\right]\right) A_{q}}{P_{q}\left(\lambda-E_{\alpha}\right)}=\frac{P_{\alpha}\left[\tilde{\alpha}_{1}\right] E_{\alpha} A_{q} \lambda}{\left(P_{q} \lambda+\tau\right)\left(\lambda-E_{\alpha}\right)}
$$

443 These equations are valid provided $\lambda \neq-\tau / P_{q}$ and $\lambda \neq E_{\alpha}(\chi) \forall \chi$. The first is a

444 single point, which can be ignored. The second exception requires $\lambda>\max _{\chi}\left(E_{\alpha}(\chi)\right)$,

445 which is simply the condition that $\lambda$ is greater than the supremum of the continuous

446 spectrum, which again we denote by $c$. Therefore, we consider (3.18) and (3.19) only

447 for $\lambda \in(c, \infty) \backslash\left\{-\tau / P_{q}\right\}$.

$448 \quad$ Applying the operator $P_{\alpha}$ to (3.19) leads to an equation for $\lambda$ :

449

450

$$
F(\lambda) \equiv P_{\alpha}\left[\frac{E_{\alpha} A_{q} \lambda}{\left(P_{q} \lambda+\tau\right)\left(\lambda-E_{\alpha}\right)}\right]-1=0
$$




\section{Miranda C. Holmes-Cerfon and J. A. Whitehead}

If $\tau=0$ this equation is exactly the constant-pressure case mentioned above. For

453 other values of $\tau$ we solved this equation numerically for $\lambda$ in $\left(q_{i}, \tau\right)$ parameter space.

454 The full regions of stability/instability and oscillating solutions for a representative value

455 of dimensionless temperature constant $T_{n}=10$ are summarized in Figure 6b. Let us

456 describe these in more detail.

457 Consider a fixed $q_{0}$ such that it is less than the flux $q_{c}$ that minimizes pressure

458 drop. If $\tau=0$ there is one eigenvalue, and the tube is unstable. As $\tau$ increases, there is

459 a critical value of $\tau$ at which a bifurcation occurs and the system has 3 eigenvalues. One

460 of these is real and the other two are complex with non-zero imaginary parts. The real

461 root is always negative and less than $-\tau / P_{q}$, so we track the signs of the complex roots

462 in order to detect instability. As $\tau$ increases, the real parts of the roots decrease,

463 eventually crossing zero so the system becomes stable. As $\tau$ is further increased, the

464 complex eigenvalues eventually disappear.

465 For $q_{0}>q_{c}$ the system is always stable. As $\tau$ increases, a similar bifurcation

466 occurs, with complex eigenvalues appearing for large $\tau$ and disappearing for even larger

$467 \tau$. For a fixed value of $\tau$, this means that there is a critical value of $q_{0}$ below which the

468 system is unstable, and above which the system is stable. This critical value is plotted

469 with diamonds in Figure 6a for several values of $\tau$. The figure shows the critical value

470 decreases as $\tau$ increases, so that the range of stable steady states is much greater with

$471 \quad$ large $\tau$. As $\tau \rightarrow \infty$, all steady states become stable, corresponding to case (i) with

472 constant flux. As anticipated, the value of $\tau$ serves the function of interpolating between

473 constant pressure drop and constant flux for quantifying a stability criterion. 


\subsection{Numerical Simulations of stability}

476

477 Numerical simulations of the nondimensional equations (3.15) were performed to

478 test the linear stability predictions. The pressure difference was either kept constant, or

479 varied according to (3.15b), and the tube radius was stepped forward in time using

480 (3.15a). Time derivatives were calculated using forward Euler, the trapezoidal rule was

481 used for integration, and 1000 eigenvalues were used to calculate the heat flux and steady

482 profiles. 40 points were used to represent the tube in the horizontal. The simulations were

483 stopped if the tube froze shut, i.e. when $\alpha(\chi, t)=0$ for some $\chi$. The numerical

484 simulations confirm the theoretical predictions. Small perturbations to a profile that is

485 linearly stable return to the original state, whereas perturbations to a profile which is

486 linearly unstable eventually freeze shut for $\tau \neq 0$. The perturbation oscillates about the

487 steady-state as it grows or decays exactly where linear theory predicts complex

488 eigenvalues.

489 Consider now the fixed-pressure case, $\tau=0$, which is unique as it has two

490 possible steady-states, one stable and the other unstable. Figure 7 shows the two different

491 types of evolution that are possible if we start with the linearly unstable profile and

492 perturb it a little. If the perturbation is mostly positive, in the direction of the stable

493 profile corresponding to the same value of $\Delta p$, then the tube opens up, and moves to the

494 stable profile. If the perturbation is mostly negative, away from the stable profile, then

495 the tube freezes shut. As the tube moves from one profile to another, its shape is always 
496 close to that of a steady profile. Any localized disturbances to the profile are rapidly

497 ironed out. This is consistent with the linear theory, which predicts large negative

498 eigenvalues in the continuous spectrum that appear to be associated with highly localized

499 eigenfunctions.

Figure 8 shows two cases of the radius at the endpoint of the tube $\alpha(1)$ in the

501 case of a growing or decaying oscillating solutions. The time constant $\tau$ was kept

502 constant, and the flux varied so that it was to the right of the critical flux in one case, and

503 to the left in the other. In the first case, a small perturbation oscillated about the steady-

504 state and eventually decayed, leaving a steady-state tube in its wake. In the second case, a

505 small perturbation oscillated about the steady-state but grew larger, and eventually the

506 tube froze shut.

\subsection{Application: length of a lava tube}

One motivation for this study was to explain the length of lava tubes observed in

511 some volcanic flows on Earth and Mars, where tubes of 50-200km have been found

512 [Sakimoto and Zuber 1998]. Such steady-state tubes, which are formed when highly

513 viscous lava flows down low-angle slopes, often terminate because of geographical

514 features such as an abrupt change in slope or reaching an ocean, and it would be

515 interesting to know whether there are physical constraints governing their lengths as well.

516 Therefore, as a final note, we would like to show some simple calculations to illustrate

517 how this model can be used to provide an upper bound for the length of a melt conduit in

518 an Earth or planetary context. In many tubes, the pressure at the upstream end of the tube 
519 is dominated by the hydrostatic pressure so we use this as the constraint. Recalling that

520 the non-dimensional pressure drop must be greater than a critical value in order for a

521 steady-state tube to exist, the length satisfies

$$
\frac{\Delta \mathrm{Pr}_{0}^{4}}{4 \kappa \mu L^{2}} \geq \Delta p_{c}\left(T_{n}\right) \quad \Leftrightarrow \quad L \leq \sqrt{\frac{\Delta \mathrm{Pr}_{0}^{4}}{4 \kappa \mu \Delta p_{c}\left(T_{n}\right)}} .
$$

523 Using typical lava parameters [Keszthelyi 1993, Sakimoto and Zuber 1998]

$524 \kappa=10^{-7} \mathrm{~m}^{2} / \mathrm{s}, \rho=2300 \mathrm{~kg} / \mathrm{m}^{3}, \mu=60(54-160) \mathrm{Pa} \cdot \mathrm{s}, T_{i}=1133-1187{ }^{\circ} \mathrm{C}$,

$525 T_{s}=1077^{\circ} \mathrm{C}, T_{0}=30^{\circ} \mathrm{C}$, (these temperatures correspond to $T_{n}=8-20$ ), and

526 calculating the hydrostatic pressure difference as $\Delta P=\rho g H$, where $H$ is the total

527 vertical distance travelled by the lava tube and $g$ is gravity, we find that a tube with a

528 radius of $10 \mathrm{~m}$ which drops $1 \mathrm{~km}$ can have a maximum length of $110-440 \mathrm{~km}$.

529 It is encouraging that this is consistent with observations, but we note that there are many

530 reasons (not detailed here) why this model is too idealized to make direct conclusions

531 about lava tubes. We note also that our mixed upstream condition applies in certain

532 flows, such as when the lava tube drains from a lava lake or an interior elastic magma

533 chamber, each receiving lava either steadily or impulsively from a source inside the earth,

534 in which case the linearized version of the geophysical upstream condition is similar to

$5353.15 \mathrm{~b}$. However, due to the difficulty of obtaining accurate data for such flows we prefer

536 not to speculate on numerical values at present. 
542 through a tube whose boundary is held below freezing, solid material forms on the

543 boundary, leaving an inner tube of flowing liquid. As the flow rate is progressively

544 decreased, the pressure drop across the tube first decreases and then increases before

545 finally the tube freezes shut.

546 We investigate a theoretical model for low-Reynolds number flow through a tube

547 with solidification, in which we solve for the shape of a steady-state tube as a function of

548 distance downstream and find the relationship between pressure drop and flux in steady-

549 state. This shows that the pressure drop has a minimum as flux is varied. The linear

550 stability of the steady-states depend on the upstream boundary condition: when constant

551 flux is applied, all states are predicted to be stable; when constant pressure drop is

552 applied, those corresponding to a flux less than the flux at the minimum are unstable, and

553 for a coupled condition the critical flux for stability is in between. In the experiments,

554 pressure drop and flux were coupled by the measuring device, so these qualitative results

555 may explain the experimental rise in pressure drop as flux is slowly decreased to freezing

556 value.

557 Attempts to produce a full quantitative comparison between the laboratory

558 experiment and the theory have produced poor results which we attribute to numerous

559 possible causes of uncertainly in the experiment. There was, of course, uncertainty in the

560 mean values as well as internal variations of viscosity and specific heat, which makes

561 quantitative comparison difficult. There is also an overall sensitivity of the system to the

562 precise tube geometry, which is not captured by an axisymmetric model. Our experiments

563 showed that small perturbations near the endpoint could initiate large-scale freezing 
564 events. Most solidifying materials have some crystal structure that might generate local

565 flaws, and even small bits of foreign material (particles, dust, microbubbles, etc.) might

566 produce effects that get magnified near the exit. It is possible that experiments using pure

567 filtered or distilled water that is completely free of dust, particles and dissolved air, could

568 produce results much closer to theory since it has very well-known material properties

569 and minor viscosity changes near freezing. However, it is important to note again that the

570 earlier experiments with water [e.g. Zerkle and Sunderland, 1968 Mulligan and Jones,

5711976 Hirata and Ishihara 1985, Weigand et al., 1997] exhibited wave formation in the

572 ice and that such local features might be common and that their role in freeze-up is

573 probably not yet fully appreciated. To clarify such points, optical views of the liquid tube

574 interiors would be very useful.

575 Overall, our findings suggest that the distance traveled by fluid in a melt conduit

576 is very sensitive to the conditions that govern pressure and flow rate at the upstream end.

577 One of our motivations was to study the paths of magma and lava flows, which are well

578 known to be quite complicated. We suggest that the sensitive interrelation between

579 upstream pressure and the stability of the tube at the downstream end, where it is most

580 likely to freeze shut, is one mechanism responsible for such complexity.

\section{Acknowledgements}

Support was received from the Geophysical Fluid Dynamics Program, which is

584 supported by the Ocean Sciences Division of the National Science Foundation under

585 Grant OCE-0325296, and from the Oceanography Section of the Office of Naval

586 Research under Grant N00014-07-1-0776. The laboratory experiments were supported by 
587 the Deep Ocean Exploration Institute of W.H.O.I. M.C. Holmes-Cerfon would like to

588 thank Lou Howard for many helpful conversations during the GFD summer program. We

589 are also very grateful for the thorough help and comments of two anonymous referees.

\section{References}

592

Bindhu, C. V., S. S. Harilal, V. P. N. Nampoori, and C. P. G. Vallabhan (1998),

594 Thermal diffusivity measurements in organic liquids using transient thermal lens

595 calorimetry. Opt. Eng. 37 (10), 2791-2794.

Bruce, P. M. and H. E. Huppert (1989), Thermal control of basaltic fissure

597 eruptions. Nature 342, 665-667.

Bruce, P. M. and H. E. Huppert (1990), Solidification and melting in dykes by the

599 laminar flow of basaltic magma, in Magma Transport and Storage, M. P. Ryan, Ed. pp.

600 87-102. Wiley, New York, 420 pp.

601 Dragoni, M., F. Donza, and A. Tallarico, (2002), Temperature distribution inside

602 and around a lava tube. Journal of Volcanology and Geothermal Research 115, 43-51.

603 Epstein M. and F. G. Chueng, (1983), Complex freezing-melting interfaces in

604 fluid flow Ann. Rev. Fluid Mech. 15, 293.

605 Graetz, L. (1883), Uber die Wärmeleitungsfähigkeit von Flüssigkeiten, Annalen

606 der Physik und Chemie 18, 79.

607 Hirata, T and M. Ishihara (1985) Freeze-off conditions of a pipe containing a flow 608 of water, Int. J Heat Mass. Trans. 28, \#2, 331-337.

609 Holmes, M. (2007), Length and shape of a lava tube. Woods Hole 
610 Oceanographic Institution Geophysical Fluid Dynamics Program Proceedings Volume

611 2007. http://www.whoi.edu/page.do?pid=19276.

612 Keszthelyi, L. P. (1993), Emplacement of the 75-km-long Carrizozo lava flow

613 field, south-central New Mexico, Journal of volcanology and geothermal research 59, $614 \quad 59-75$.

615 Klingelhofer, F., M. Hort, H. J. Kumpel, and H. U. Schmincke, (1999),

616 Constraints on the formation of submarine lava flows from numerical model calculations.

617 Journal of Volcanology and Geothermal Research 92, 215-229.

618 Lee D. G. and R. D. Zerkle (1969), The effect of liquid solidification in a parallel

619 plate channel upon laminar-flow heat transfer and pressure drop. J. Heat Transfer, 91, $620 \quad 583-585$.

621 Lister, J. and Dellar, P. (1996), Solidification of pressure-driven flow in a finite 622 rigid channel with applications to volcanic eruptions. J. Fluid Mech., 323, 267-283.

623 Mulligan J. C. and D. D. Jones, (1976), Experiments on heat transfer and pressure 624 drop in a horizontal tube with internal solidification. Int. J. Heat and Mass Transfer 19, $625 \quad 213-219$.

626 Richardson, S. M. (1985), Injection moulding of thermoplastics: freezing of at 627 gates, Rheol. Acta, 24, 497-508.

628 Richardson, S. M. (1986), Injection moulding of thermoplastics: Freezing of 629 variable-viscosity fluids. III Fully developed flows, Rheol. Acta, 25, 372-379.

630 Sakimoto, S. E. H. and M. T. Zuber (1998), Flow and convective cooling lava 631 tubes. J. Geophys. Res . 103, 27465-27487.

632 Sakimoto, S. E. H. and T. K. P. Gregg (2001), Channeled flow: Analytic 
633 solutions, laboratory experiments, and applications to lava flows. J. Geophys. Res. 106, $634 \quad 8629-8644$.

635 Sampson P. and R. D. Gibson (1981), A mathematical model of nozzle blockage 636 by freezing, Int J. Heat and Mass Transfer. 24. 231-241.

637 Turcotte, D. L. and G. Schubert, (2002) Geodynamics. Cambridge University 638 Press. 482 pp.

639 Weigand B., J. Braun, S. O. Neumann, and K. J. Rinck, (1997) Freezing in forced 640 convection flows inside ducts: a review, Heat and Mass Transfer, 32, 341-351.

641 Zerkle, R. D. and J. E. Sunderland 1968, The effect of liquid solidification in a 642 tube upon laminar-flow heat transfer and pressure drop. J. Heat Transfer, Trans. ASME 643 Series C, 90, 183-190. 


\section{Tables}

\begin{tabular}{|c|c|}
\hline$A$ & Upstream reservoir cross sectional area \\
\hline$A_{n}$ & Coefficients of temperature solution \\
\hline$E$ & Radial heat flux in the solid \\
\hline$G_{n}$ & Coefficients of solution for flux of temperature $A_{n} \phi_{n}{ }^{\prime}(1)$ \\
\hline$H$ & Elevation of liquid upstream of the tube \\
\hline$I$ & Radial heat flux in the liquid \\
\hline$L$ & Tube length \\
\hline$L_{H}$ & Latent heat of solidification \\
\hline$P$ & Pressure \\
\hline$\Delta P$ & Pressure drop across entire tube \\
\hline $\operatorname{Pr}$ & Prandtl number \\
\hline$Q$ & Volume flux through the tube \\
\hline$Q_{i}$ & Volume flux into upstream reservoir \\
\hline$S$ & Stefan number $L_{H} / C_{p}\left(T_{i}-T_{s}\right)$ \\
\hline$T$ & Temperature in the liquid \\
\hline$T_{e}$ & Temperature in the solid \\
\hline$T_{i}$ & Temperature of fluid at inlet \\
\hline$T_{n}$ & $\begin{array}{l}\text { Dimensionless temperature constant, equal to } \\
\left(T_{s}-T_{0}\right) /\left(T_{i}-T_{s}\right)\end{array}$ \\
\hline$T_{0}$ & Temperature at the outer radius \\
\hline$T_{s}$ & Temperature of solidification \\
\hline$a$ & Radius of the solid-liquid interface \\
\hline$c_{p}$ & Specific heat \\
\hline$g$ & Acceleration of gravity \\
\hline$k$ & Thermal conductivity \\
\hline$p$ & Dimensionless pressure \\
\hline$\Delta p$ & Dimensionless pressure drop $r_{0}^{4} \Delta P / 4 \mu \kappa L^{2}$ \\
\hline$\Delta p_{c}\left(T_{n}\right)$ & $\begin{array}{l}\text { Critical value of pressure drop, below which no steady-state } \\
\text { tube is possible }\end{array}$ \\
\hline$q$ & Dimensionless flux $2 Q / \kappa \pi L$ \\
\hline$q_{i}$ & Dimensionless inlet flux $2 Q_{i} / \kappa \pi L$ \\
\hline$q_{c}\left(T_{n}\right)$ & Critical value of flux, at which $\Delta p=\Delta p_{c}$ \\
\hline$r$ & Radial coordinate \\
\hline$r_{0}$ & Outer tube radius \\
\hline$t$ & Time \\
\hline$u$ & Fluid velocity \\
\hline$u^{\prime}$ & Dimensionless fluid velocity \\
\hline$v$ & Velocity in a radial direction \\
\hline$x$ & Coordinate along the axis of the tube \\
\hline$\alpha$ & Dimensionless radius of solid-liquid interface $a / r_{0}$ \\
\hline
\end{tabular}




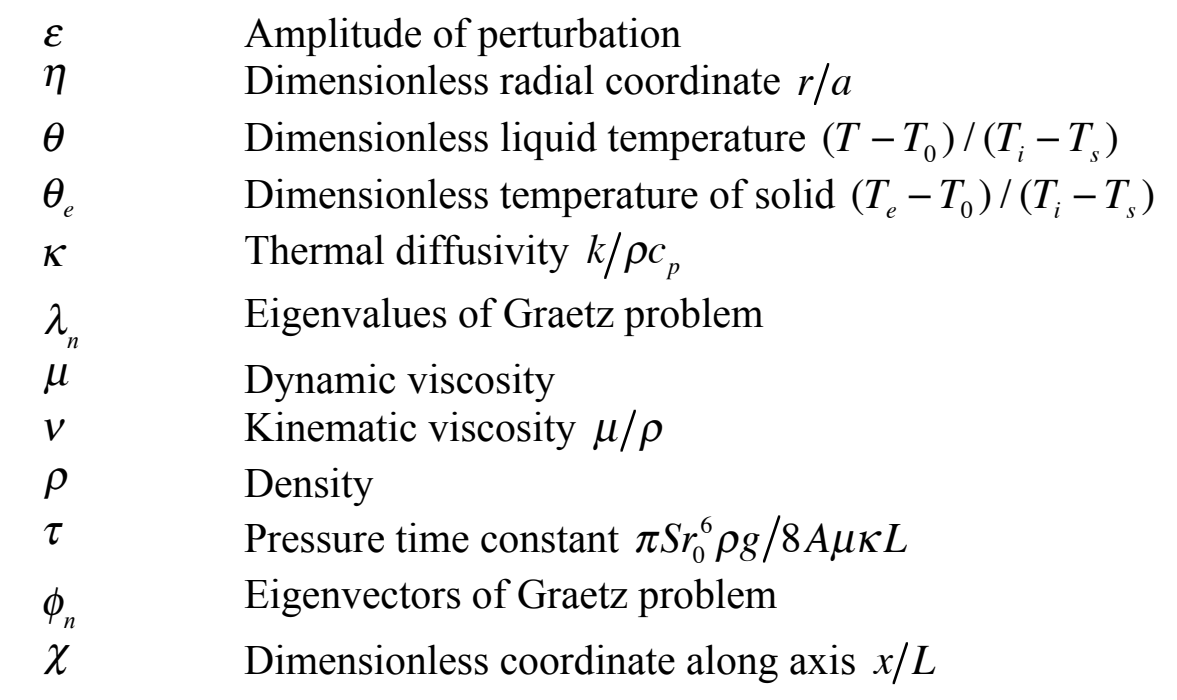

645

646

647

648
Table 1 List of symbols. For perturbation theory, the basic state is denoted by subscript 0 and the perturbation by subscript 1 .

$$
\begin{aligned}
& E(\alpha(\chi, t))=\frac{\partial \theta_{c}}{\partial \eta}=\frac{T_{n}}{\ln \alpha} \\
& I(\chi, q)=\frac{\partial \theta}{\partial \eta}=\sum G_{n} e^{-\lambda_{n}^{2} \chi / q} \\
& \left.E_{\alpha}(\chi) \equiv \frac{1}{\alpha_{0}} \frac{d E}{d \alpha}\right|_{\alpha_{0}}=\frac{-T_{n}}{\alpha_{0}^{2}\left(\ln \alpha_{0}\right)^{2}}
\end{aligned}
$$

649

$$
\begin{aligned}
& \left.P_{q} \equiv \frac{\partial \Delta p}{\partial q}\right|_{\alpha_{0}, q_{0}}=\int_{0}^{1} \frac{1}{\alpha_{0}^{4}} d \chi \\
& \left.P_{\alpha}\left[\alpha_{1}\right] \equiv \frac{\delta \Delta p}{\delta \alpha}\right|_{\alpha_{0}, q_{0}}\left[\alpha_{1}\right]=q_{i} \int_{0}^{1} \frac{-4 \alpha_{1}}{\alpha_{0}^{5}} d \chi \\
& \left.A_{q} \equiv \frac{\partial \alpha_{0}}{\partial q}\right|_{q_{0}}=\alpha_{0} \frac{T_{n}}{\left(\sum G_{n} e^{-\lambda^{2} \chi / q_{i}}\right)^{2}} \sum G_{n} \frac{\lambda_{n}^{2} \chi}{q_{i}^{2}} e^{-\lambda^{2} \chi / q_{i}}
\end{aligned}
$$

Table 2. Particular form of the functions used in the perturbation calculations. 